\title{
MULTI-LAYER WS2 AND MOS2 BASED PLASMONIC SOLAR CELL FOR SMART ENERGY HARVESTING.
}

\author{
Z.Oumekloul ${ }^{1,2, *}$ Y. Achaoui ${ }^{1}$, A.Mir $^{1}$, A.Akjouj $^{2}$ \\ ${ }^{1}$ Laboratory for the Study of Advanced Materials and Applications (LEM2A), Physics Department, Moulay Ismail University of Meknes, \\ B.P. 11201, Zitoune, Meknes, Morocco. \\ ${ }^{2}$ Institute of Electronics, Microelectronics and Nanotechnology, CNRS-8520, Department of Physics, FST, University of Lille, \\ 59652 Villeneuve d'Ascq, France.
}

KEY WORDS: Localized Surface Plasmon Resonance, gold nanowires, 2D materials, transition metal dichalcogenides, sensing, solar cell.

\begin{abstract}
:
One of the best challenges regarding the futuristic vision of smart-city technologies is to offer a comfortable self-governance energy, especially when it comes to electricity storage. If one wants to revolutionize a pre-existing way of living, it is inescapable to neglect basic ingredients gathered from basic physics. The topic of Metamaterials represents a key field that might be explored and thus exploited to propose unprecedented ideas for completely no-existing properties and functionalities. Unlike other ambitious techniques, with a simple stratified surface in combination with a suitable choice of materials, it is possible to propose new solar cells operating in a broad range of frequencies. In this paper, we demonstrate a manner to achieve strong coupling interaction between metallic gold nanowires with a $W S_{2}$ and $M o S_{2}$ multi-layer. The novelty of this work lies in the drastic stability of the effect of the thickness layer variation on both, absorption performances and the electric field distribution within the visible and near-infrared range. Accordingly, this new design may be considered of prime importance in several areas such as sensing and solar cell efficiency, to cite a few examples.
\end{abstract}

\section{INTRODUCTION}

Surface Plasmons (SPs), a collective oscillation of free electrons in the conduction band of metals, are evanescent waves sensitive to variation the optical properties of the surrounding media. It is well known that a p-polarization can cause a charge creation at the metallic interface and excite the SPs. This phenomenon, generally referred to as Surface Plasmon Resonance (SPR), takes place when the necessary momentum of the incident light is provided through a prism [1]. Under these searched conditions, a maximum energy of the TM-polarized light gets transferred to the electron and results in a sharp peak in the absorption curve. This reality gives rise to the plasmonic absorbers based on metallic nanostructures' designs. In the last few years, this physics has been used to create the next generation of solar cells as a smart solution in the race to a smart energy harvesting.

Localized surface plasmons resonance (LSPR) is a family of SPs associated with metal particles of dimensions much smaller than the wavelength of the radiation. The underlying physics of the aforementioned LSPR opened a new avenue to design metallic nanostructures, which attract a great interest thanks to their present and future potential applications. Since then, plasmonic absorbers has undergone a great development and has found various applications ranging from biosensors [2], dedicated to proteins and antibodies label-free detection,

opto-electronic devices, to efficiency improvement of photovoltaic cells [3]. Moreover, the study of several physical parameters of the nanostructures makes it a promising assess of the next generation of absorbing surfaces. Parameters, such as the substrate's chemical composition upon which the metal is deposited, the particles' size and form, as well as the surrounding environment, can be optimized and evaluated using numerical models to predict their effects. Furthermore, these models allow the exploitation of new materials such as graphene and 2D Transition dichalcogenide (TMDs) as Molybdenum disulfide $\left(M o S_{2}\right)$ and Tungsten Disulfide $\left(W S_{2}\right)$ [4,5,6].

Interaction of nanoparticles with 2D monoatomic thickness materials such as $W S_{2}$ offers further perspectives for optoelectronic applications. In sub-nanometric distances, other effects come into the equation, as a result of the tunneling effect of electrons between the metallic nanoparticles and the active semiconducting layer. $W S_{2}$ is a two-dimensional nanomaterial with a hexagonal crystal lattice, in which atoms are jointed by strong bonding forces and layers combination through the Van der Waals forces. From a plasmonic point of view, $W S_{2}$ presents favorable optical and tribological properties among others. Firstly, owing to the electron distribution, $W S_{2}$ has tribological properties including a state of near zero friction or super lubricity. Secondly, $W S_{2}$ is thermally more stable and resilient to oxidation. Last but not least, the nano-scale $W S_{2}$ has a high density, small size, large specific surface area and a high specific surface energy.

Recently, many studies[5-8] have been conducted to shown that the integration of these monoatomic coating above the substrate revealed a significant enhacement of the optical properties such as absorption, light emission and photoluminescence.

In this work, we report on a numerical study using the Finite Elements Method (FEM) and the Drude-Lorentz model to predict accurately the optical signature of a gold nanowires anchored onto $W S_{2}$ or $M o S_{2}$ surfaces. In our previous work, we investigated the way that different parameters of the proposed gold nanowires affect the absorption enhancement. As a result, we carried out the best structuration for absorption enhancement. We investigate in this paper conditions underwhich periodicity affects the absorption and also how the effect of periodicity on the absorption. A particular attention is given to the influence 
of $W S_{2}$ and $M o S_{2}$ on the performances. By studying several $W S_{2}$ and $M o S_{2}$ thickness parameters, we highlighted the optimum configuration trade-off between maximum absorption and frequency range operation.

\section{THEORETICAL MODEL}

Calculations are performed using the finite element method (FEM), which solves Maxwell's equations by discretizing space. Our calculations are performed in a two-dimensional (2-D) box (along $\mathrm{x}$ and $\mathrm{y}$ axes) with a propagation along the $\mathrm{y}$ axis. Perfectly Matched Layers (PML) conditions are applied at the boundaries towards y-direction, to avoid spurious reflections of outgoing waves. Along the $\mathrm{x}$ direction, the unit cell is repeated periodically (see Figure 1) and the structure is supposed to be infinite along the $\mathrm{z}$ direction. Space is discretized in both $\mathrm{x}$ and $\mathrm{y}$ directions using a triangular mesh. The input electromagnetic field, having TM polarization, is generated at the top part of the unit cell by a current source parallel to the y axis. The transmitted signal, probed at the end of the bottom part of the unit cell, is recorded as a function of frequency. All the absorption spectra are normalized with respect to the problem.

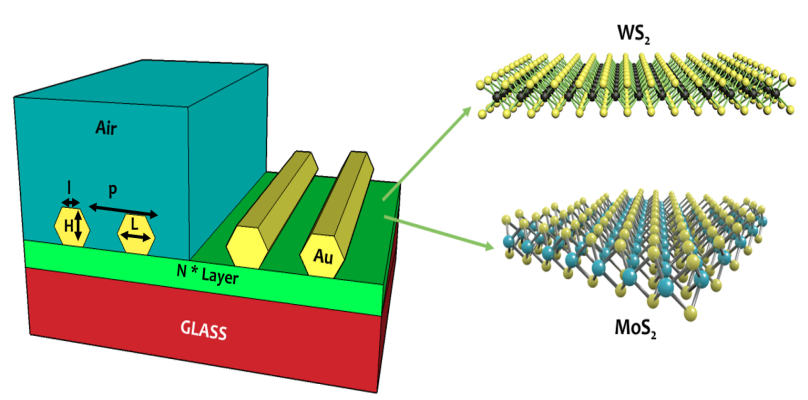

Figure 1. 3D nanostructure with TMDs layer.

The plasmonic nanostructre studied in this work is constructed by gold nanowires with hexagonal shape. The hexagonal gold nanowire array used here is characterized by its height $\mathrm{H}$, the two diameters $\mathrm{L}$ and $\mathrm{l}$, as well as the period $\mathrm{p}$ (see Figure 1). This hexagonal nanowire periodic structure is arranged on a $\mathrm{N}$ layers of $W S_{2}\left(d_{W S_{2}}=\mathrm{N}^{*} 0.8 \mathrm{~nm}\right)$ or $M o S_{2}\left(d_{M o S_{2}}=\mathrm{N}^{*} 0.65\right.$ $\mathrm{nm}$ ) in order to understand the effect of these two TMDs materials on the absorption enhancement. Finally, the two elements are formed on a glass surface (Figure 1). In the study, we take into account a normal incidence of the electromagnetic field as a type of excitation, and we vary the wavelength in the visible range in order to detect the LSPR in the metal structure by modifying the periodicity and the thickness of the TMDs materials. The thickness of different $W S_{2}$ or $M o S_{2}$ layers are given in Table 1.

\begin{tabular}{|c|c|}
\hline$W S_{2}$ & $M o S_{2}$ \\
$\widehat{n}=4.9+0.3124 \mathrm{i}$ & $\widehat{n}=5.08+1.1723 \mathrm{i}$ \\
\hline $1 \mathrm{~L}=0.8 \mathrm{~nm}$ & $1 \mathrm{~L}=0.65 \mathrm{~nm}$ \\
\hline $2 \mathrm{~L}=1.6 \mathrm{~nm}$ & $2 \mathrm{~L}=1.3 \mathrm{~nm}$ \\
\hline $3 \mathrm{~L}=2.4 \mathrm{~nm}$ & $3 \mathrm{~L}=1.95 \mathrm{~nm}$ \\
\hline
\end{tabular}

Table 1. Parameters of $W S_{2}$ and $M o S_{2}$ layers[9].

Employing the optical microscopy Castellanos-Gomez et al.[10] have quantitatively analyzed the data to determine the thickness and refractive index using different illumination wavelengths in the visible spectrum. Here, we suppose that the expressions of refractive index are constant in this range since it does not significantly influence the plasmonic mode. The dielectric constant of metal $(\mathrm{Au})$ is described by the Lorentz-Drude model. For frequency-dependent complex permittivity of metal (gold), the formula is given by [11]:

$$
\varepsilon_{D L}(\omega)=\varepsilon_{\infty}+\sum_{m=0}^{M}\left(\frac{G_{m} \Omega_{m}^{2}}{\omega_{m}^{2}-\omega^{2}+i \omega \Gamma_{m}}\right)
$$

where $\varepsilon_{\infty}$ is the relative permittivity at infinite frequency, $\Omega_{m}$ is the plasma frequency, $\omega_{m}, f_{m}$, and $\Gamma_{m}$ are, respectively, the resonance frequency, the strength, and the damping frequency of the oscillator. The Lorentz-Drude model uses $\mathrm{M}=5$ damped harmonic oscillators to describe the small resonances observed in the metal frequency response. The values of constants in Eq. 1 are given in Table 2. This model allows us to fit experimental data for the frequency-dependent dielectric constant of metals including both real and imaginary parts, with good agreements in the visible wavelength range $(400<\lambda<800 \mathrm{~nm})$.

\begin{tabular}{|c|c|c|c|c|}
\hline Oscillateur $\mathrm{m}$ & $G_{m}$ & $\omega_{p}\left(\right.$ rad. $\left.^{-1}\right)$ & $\omega_{m}\left(\right.$ rad.s $\left.^{-1}\right)$ & $\Gamma_{m}\left(\right.$ rad. $\left.^{-1}\right)$ \\
\hline 0 & 0.76 & $1.37188 .10^{16}$ & 0 & $8.05202 .10^{13}$ \\
\hline 1 & 0.024 & $1.37188 .10^{16}$ & $6.30488 .10^{14}$ & $3.66139 .10^{14}$ \\
\hline 2 & 0.01 & $1.37188 .10^{16}$ & $1.26098 .10^{15}$ & $5.24141 .10^{14}$ \\
\hline 3 & 0.71 & $1.37188 .10^{16}$ & $4.51065 .10^{15}$ & $1.32175 .10^{15}$ \\
\hline 4 & 0.601 & $1.37188 .10^{16}$ & $6.53885 .10^{15}$ & $3.78901 .10^{15}$ \\
\hline 5 & 4.384 & $1.37188 .10^{16}$ & $2.02364 .10^{16}$ & $3.36362 .10^{15}$ \\
\hline
\end{tabular}

Table 2. The gold parameter values for Drude-Lorentz model[11].

\section{NUMERICAL RESULTS}

To keep the shape of our hexagonal structure, parameter L must be larger than 1 . Thus, we took in this case $L=1+5 \mathrm{~nm}$. The geometrical parameters of the hexagonal nanowire are defined by the experimental and numerical works found in the literature $[12,13,14]$ and the values chosen are: $1=35 \mathrm{~nm}, \mathrm{~L}=40 \mathrm{~nm}$ and $\mathrm{H}=15 \mathrm{~nm}$.

\subsection{The influence of the periodicity}

It is well known in plasmonics that periodicity of the plated gold nanowires affects its optical signature. Thus, we studied as a first work the absorption of several periodicity with values of $\mathrm{p}=50: 10: 90 \mathrm{~nm}$ to understand the influence of the periodicity $\mathrm{p}$ on the plasmonic resonance. Figure 2 shows the obtained results. It can be seen that the best value is obtained for $\mathrm{p}=50 \mathrm{~nm}$. It is therefore possible to notice the influence of the geometry of the structure, by varying $\mathrm{p}$, one can easily observe the effect on the absorption spectrum for a TM polarization. Figure 2 presents the absorption coefficient versus the wavelength. Gradually, as the particles approach each other, the optical behavior of structure becomes increasingly interacting; when the particles approach, $\mathrm{p}=50 \mathrm{~nm}$, it shows a strong absorption of incident energy (77\%). The absorption enhancement of the periodicity is given from $42 \%(\mathrm{p}=90 \mathrm{~nm})$ to $77 \%$ $(\mathrm{p}=50 \mathrm{~nm})$. When the inter-particle distance is small, the mutual influence of particles has a strong coupling. Thus, this fact results in an electric field enhancement that can be explained by the fact that the electric field is resulting from the sum of the incident field and the resonance between the nanowires. This underliying physics explains our choice of the parameter $\mathrm{p}=50 \mathrm{~nm}$. 


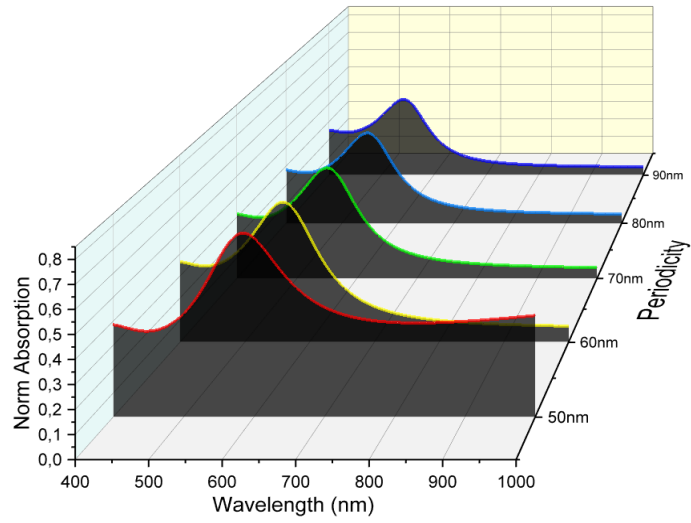

Figure 2. Evolution of the absorption coefficient of an hexagonal gold nanowires along with $\mathrm{p}$.

Let us mention that when $\mathrm{p}$ vary from $50 \mathrm{~nm}$ to $90 \mathrm{~nm}$ the resonant wavelength decrease and shift to step to the blue range. These results are confirmed with exprimental works in the literature for the same systemes[12]. For low coupling regimes between the metallic nanostructures, $\mathrm{p}$ variation has almost no effect on the response of the particles. So, compared to other plasmonic structures considered in the literature [12-15] as the gold ribbon a better result can still be seen. This plasmonic structure concentrates and absorbs the field in a very subwavelength volume, which represents a crucial interest for plasmonic solar cells.

\subsection{The influence of the TMDs active layers}

Based on the results in Figure 2, it can be seen that the periodicity has an impact on the absorption in which we performed an enhancement from $42 \%$ to $77 \%$.

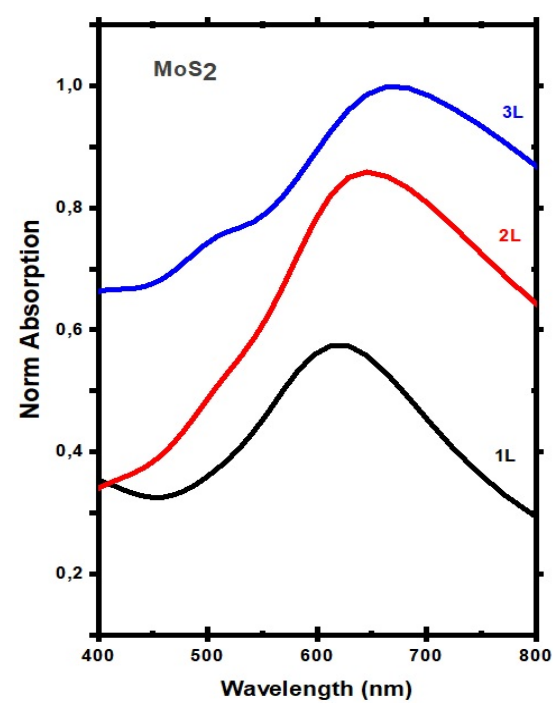

Figure 3. Variation of normalized Absorption along with Wavelength for several $\mathrm{MoS}_{2}$ layers.

In this section, we added the potentials TMDs materials as a spacer between glass and nanowires as they are promising materials in Plasmonics. For the $W S_{2}$ and $M o S_{2}$ thickness layers, the geometrical values are grouped in Table 1 . As shown in Figure 3, for $\mathrm{MoS}_{2}$, the absorption coefficient can reach 57\% at $612 \mathrm{~nm}$ for 1 layer to $99 \%$ at $663 \mathrm{~nm}$ for 3 layers. Thus, we can see a red shift of $51 \mathrm{~nm}$.
In addition, as shown in Figure 4, for $W S_{2}$, the absorption coefficient can reach $58 \%$ at $628 \mathrm{~nm}$ for 1 layer to $90 \%$ at $694 \mathrm{~nm}$ for 3 layers. Hence, we can see a red shift of $66 \mathrm{~nm}$.

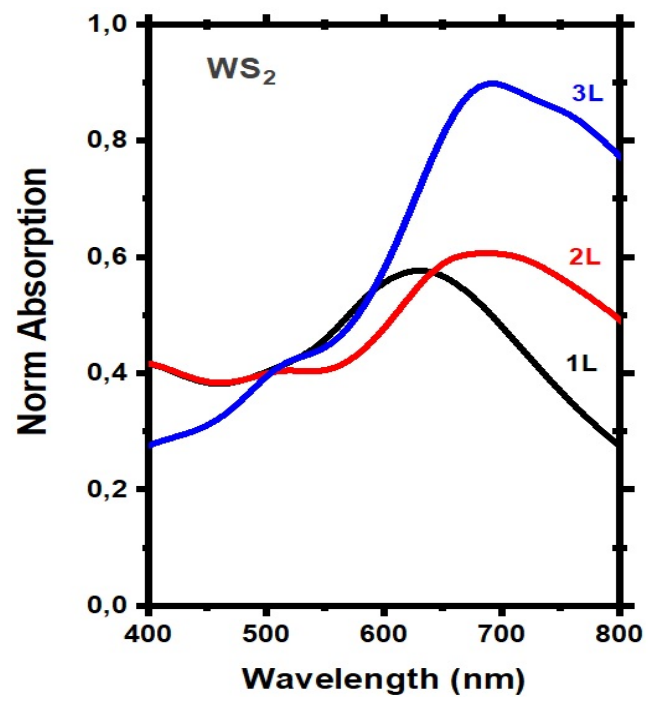

Figure 4. Variation of normalized Absorption along with Wavelength for several $W S_{2}$ layers.

From these Figures, each TMDs material presents advantages compared to the other. For $\mathrm{MoS}_{2}$, it presents a strong absorption in the case of 3 layers that can reach $99 \%$ at $663 \mathrm{~nm}$. However, the resonance shift is less important compared to $W S_{2}$ that presents a $15 \mathrm{~nm}$ shift higher and $9 \%$ less absorption. These results can be explained by the imaginary and real parts of the optical refractive index. For $\mathrm{MoS}_{2}$, the shift is less because of its higher imaginary part value compared to $W S_{2}$. However, the real part is more higher for $W S_{2}$ than the one of $M o S_{2}$. This reality explains the higher absorption that presents $\mathrm{MoS}_{2}$. This observation is confirmed by the field maps presented in Figure 5.

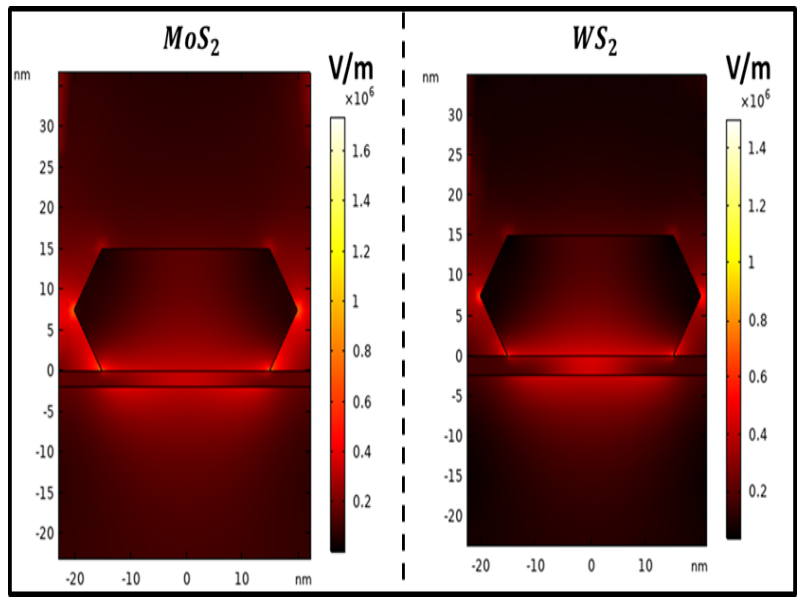

Figure 5. Electric field maps of the two TMDs materials in the case of 3 layers.

In this Figure, we can see that the nanowire presents hot spots in each corner, and especially, the two corners in contact with the TMDs active layers, which generally has losses in terms of localized field intensity. In our design, the interface between gold nanostructures and the TMDs layers allows to absorb light. This strong absorption is due to doping to the excitation of the valence electrons in TMDs layers by the strong confinement of 
the LSPR mode located in the interface of gold nanoparticles and its corners.

\section{CONCLUSION}

In this work, a theoretical simulation is done to observe the effect of two TMDs materials, which are $M o S_{2}$ and $W S_{2}$, on the absorption coefficient and electric field distribution of a new nanostructure. The proposed nanostructure is structured by a gold nanowire with hexagonal shape deposed on $\mathrm{N}$ layer of two TMDs materials. From our results, we found that $M o S_{2}$ is more desirable in the absorption purposes compared to $W S_{2}$. However, the latter presents a red shift greater than $M o S_{2}$. Physically speaking this phenomenon is due doping to the excitation of the valence electrons in TMDs layers by the strong confinement charge density of the LSPR mode located in the interface of gold nanoparticles and its corners. We have found that the proposed nanostructures based on the hexagonal gold nanowire with TMDs active layers can be a better choice for the optoelectronic devices. Significant efforts have been made experimentally in the last years to increase the optical properties in the plasmonic nanostructures, but it seems necessary to wait a few years to notice technical improvements, particularly in this new generation of solar cell.

\section{REFERENCES}

[1] Maier, S. A. Plasmonics: Fundamentals and Applications. (Springer Science Business Media, 2007).

[2] Homola, J. Piliarik, M. Surface Plasmon Resonance (SPR) Sensors. in Surface Plasmon Resonance Based Sensors (ed. Homola, J.) 45-67 (Springer Berlin Heidelberg, 2006). doi:10.1007/5346_014.

[3] Pillai, S., Catchpole, K. R., Trupke, T. Green, M. A. Surface plasmon enhanced silicon solar cells. Journal of Applied Physics 101, 093105 (2007).

[4] Tsai, M.-L. et al. Monolayer MoS2 Heterojunction Solar Cells. ACS Nano 8, 8317-8322 (2014).

[5] S. Wang et al., Limits to Strong Coupling of Excitons in Multilayer WS2 with Collective Plasmonic Resonances, ACS Photonics, vol. 6, no. 2, pp. 286-293, (Feb. 2019).

[6] Zeng, S. et al. Graphene-MoS2 hybrid nanostructures enhanced surface plasmon resonance biosensors. SENSORS AND ACTUATORS B 207, 801-810 (2015).

[7] Butun, S., Tongay, S. Aydin, K. Enhanced Light Emission from Large-Area Monolayer MoS2 Using Plasmonic Nanodisc Arrays. Nano Lett. 15, 2700-2704 (2015).

[8] Mukherjee, B. Simsek, E. Plasmonics Enhanced Average Broadband Absorption of Monolayer MoS2. Plasmonics 11, 285-289 (2016).

[9] Ouyang, Q. et al. Sensitivity Enhancement of Transition Metal Dichalcogenides/Silicon Nanostructure-based Surface Plasmon Resonance Biosensor. Sci Rep 6, 28190 (2016).

[10] Castellanos-Gomez, A., Agraït, N. Rubio-Bollinger, G. Optical identification of atomically thin dichalcogenide crystals. Appl. Phys. Lett. 96, 213116 (2010).
[11] Palik, E. D. Handbook of Optical Constants of Solids: Volume 1.(Elsevier, 2012).

[12] Oumekloul, Z., Lahlali, S., Mir, A. Akjouj, A. Evolution of LSPR of gold nanowire chain embedded in dielectric multilayers. Optical Materials 86, 343-351 (2018).

[13] Akjouj, A. et al. Nanometal plasmonpolaritons. Surface Science Reports 68, 1-67 (2013).

[14] Saison-Francioso, O., Lévêque, G., Boukherroub, R., Szunerits, S. Akjouj, A. Dependence between the Refractive-Index Sensitivity of Metallic Nanoparticles and the Spectral Position of Their Localized Surface Plasmon Band: A Numerical and Analytical Study. J. Phys. Chem. C 119, 28551-28559 (2015).

[15] Galopin, E. et al. Sensitivity of Plasmonic Nanostructures Coated with Thin Oxide Films for Refractive Index Sensing: Experimental and Theoretical Investigations. J. Phys. Chem. C 114, 11769-11775 (2010). 